\title{
Machine Learning Framework to Predict Chronic Kidney Disease using Ensemble Algorithm
}

\author{
Nikhila G, Meghashree A.C
}

\begin{abstract}
Chronic Kidney Disease (CKD) is a worldwide concern that influences roughly $10 \%$ of the grown-up population on the world. For most of the people the early diagnosis of CKD is often not possible. Therefore, the utilization of present-day Computer aided supported strategies is important to help the conventional CKD finding framework to be progressively effective and precise. In this project, six modern machine learning techniques namely Multilayer Perceptron Neural Network, Support Vector Machine, Nä̈ve Bayes, K-Nearest Neighbor, Decision Tree, Logistic regression were used and then to enhance the performance of the model Ensemble Algorithms such as ADABoost, Gradient Boosting, Random Forest, Majority Voting, Bagging and Weighted Average were used on the Chronic Kidney Disease dataset from the UCI Repository. The model was tuned finely to get the best hyper parameters to train the model. The performance metrics used to evaluate the model was measured using Accuracy, Precision, Recall, F1-score, Mathew`s Correlation Coefficient and ROC-AUC curve. The experiment was first performed on the individual classifiers and then on the Ensemble classifiers. The ensemble classifier like Random Forest and ADABoost performed better with 100\% Accuracy, Precision and Recall when compared to the individual classifiers with 99.16\% accuracy, $98.8 \%$ Precision and 100\% Recall obtained from Decision Tree Algorithm
\end{abstract}

Keywords: CKD, Machine Learning, Ensemble Algorithm, ADABoost, Bagging.

\section{INTRODUCTION}

Chronic Kidney Disease (CKD) influences $10 \%$ of the world`s population, and millions kick the bucket every year since they don't approach reasonable treatment[1]. CKD is a huge general medical problem, the tide of which proceeds to unyielding rise. According to the Global Burden of Disease Study in the year 2015, kidney disease was the $12^{\text {th }}$ most regular reason for death, representing 1.1 million deaths around the world. By and large CKD mortality has expanded

Revised Manuscript Received on May 15, 2020.

* Correspondence Author

Nikhila G*, PG Student, Department of ECE, VTU, CPGS, Mysore, Karnataka, India. Email ID: niki130782@gmail.com

Meghashree A.C, Assistant Professor, Department of ECE, VTU, CPGS, Mysore, Karnataka, India. Email ID: meghashree1988@gmail.com

(C) The Authors. Published by Blue Eyes Intelligence Engineering and Sciences Publication (BEIESP). This is an open access article under the CC BY-NC-ND license (http://creativecommons.org/licenses/by-nc-nd/4.0/) by $31.7 \%$ in the course of the most recent 10 years, making it one of the quickest rising significant reasons for death, close by diabetes and dementia[2]. In a similar report, CKD positioned as the $17^{\text {th }}$ driving reason for worldwide years loss of life, a $18.4 \%$ expansion since 2005 , and the $3^{\text {rd }}$ biggest increment of any significant reason for death. Chronic kidney Disease (CKD) implies the kidneys are harmed and cannot channel blood the manner in which they should. The infection is classified "Chronic" due to the fact that the harm to the kidneys gradually happens over a significant amount of time. This harm to the kidney can make other diseases to develop in the body. CKD can likewise cause many other medical issues[3]. Kidney disease gradually can deteriorate after some amount of time and may become main causes for the kidney to completely stop functioning. Early detection of Chronic Kidney disease is essential to protect the kidney from further damage and provide suitable treatment. The significant reasons for CKD are diabetes and hypertension which are liable for up to $66 \%$ of the CKD cases.

A few medications can cause CKD, particularly some torment killing medications (analgesics) whenever assumed control over quite a while. Frequently specialists can't figure out what caused the issue. In the beginning period there are ordinarily no indications however later, side effects like swollen ankles, tiredness, regurgitating, loss of craving, or disarray may appear. The treatment for CKD for the most part centers on easing back the movement of the kidney harm, as a rule by controlling the fundamental causes. An early finding and treatment can keep CKD from deteriorating. At the point when the results reach to the last stage, it requires customary dialysis or a kidney transplant to keep up the standard life.

\section{RELATED WORK}

Charleonnan et al.[4] built a machine learning technique for detecting the chronic kidney disease. The machine learning algorithm used were K-nearest neighbors (KNN), support vector machine (SVM), logistic regression (LR), and decision tree (DT) classifiers. These four models were built from chronic kidney disease dataset available in the UCI dataset repository. The performance of each models is compared together in order to choose the best classifier. Based on the experiment Support Vector Machine was the best algorithm with 98.3\% Accuracy, 99\% Sensitivity and 98\% Specificity.

Published By:

Blue Eyes Intelligence Engineering

\& Sciences Publication

(C) CoDvriaht: All riahts reserved.

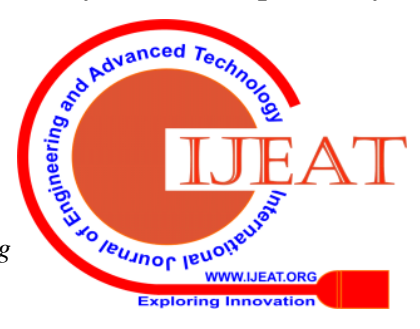


In the research conveyed by Asif Salekin [5] a innovative methodology to distinguish CKD utilizing Machine Learning strategies is used. The dataset with 400 patient records which includes 250 CKD recognized patients with 24 properties was used. The classifiers utilized are K-Nearest Neighbors (K-NN), Random Forest (RF), and neural systems to classify the patients with CKD and NoCKD.

Utilizing a wrapper technique, the number of features to discover the properties which identify CKD with high accuracy is reduced. The high accuracy was obtained using only 5 attributes. The consequences of this investigation have presented new aspects to be utilized by classifiers for distinguishing CKD all the more precisely.

The research conveyed by Pinar Yildirim [6] used Multilayer Perceptron to classify patients with Chronic Kidney Disease. The dataset used was one from the UCI dataset repository. The imbalance in the class size of the dataset was overcome by using different techniques like over sampling, SMOTE, Resample and Spread Subsample. Resample method of MLP with a learning rate of 0.3 gave a Sensitivity of $99.8 \%$ with the lowest RMSE of 0.0417 . Resample method of MLP with a learning rate of 0.3 gave a precision of $99.8 \%$ with the lowest RMSE of 0.0417.

In the investigation completed by W. Gunarathne, K. Perera, and K. Kahandawaarachchi [7], used algorithms such as Multiclass Decision Forest, Multiclass Neural Network, Multiclass Decision Jungle, Multiclass Logistic Regression for classifying patients with kidney disease and No kidney disease. The performance metrics used was accuracy. Based on accuracy Multiclass Decision Forest proved to be the best classifier with an accuracy of 99.1\%. The disadvantage of this model is the evaluation was based on only one metrics.

In 2019, J. Xiao et al. [8] conducted a study on predicting Chronic Kidney Disease using nine classification model. The Recall, Specificity, Accuracy, ROC-AUC, log loss was evaluated for each model. The linear models like lasso regression, Elastic Net, ridge regression and logistic regression gave an average AUC of $87 \%$ and a precision of $80 \%$. Logistic regression performed the best, reaching an AUC of $87.3 \%$, sensitivity and specificity of 0.83 and 0.82 , respectively.

\section{MATERIAL AND METHOD}

The proposed framework has been created with the mean to characterize individuals with Chronic Kidney Disease and No Kidney disease. The execution of several machine learning models for chronic kidney disease prediction on full were performed. The dataset used was obtained from the UCI data repository. The well-known machine learning algorithm used were Logistic Regression, K-Nearest Neighbor(K-NN), Multilayer Perceptron (MLP), Support Vector Machine (SVM), Decision Tree (DT), and Naïve Bayes (NB) were utilized in the framework. The model's approval and execution assessment measurements were processed. The performance of the base classifier was enhanced by using ensemble algorithms such as Majority Voting, Weighted Average, Bagging, ADABoost, Gradient Boosting and Random Forest. The performance of these classifiers was compared with that of the base classifiers.

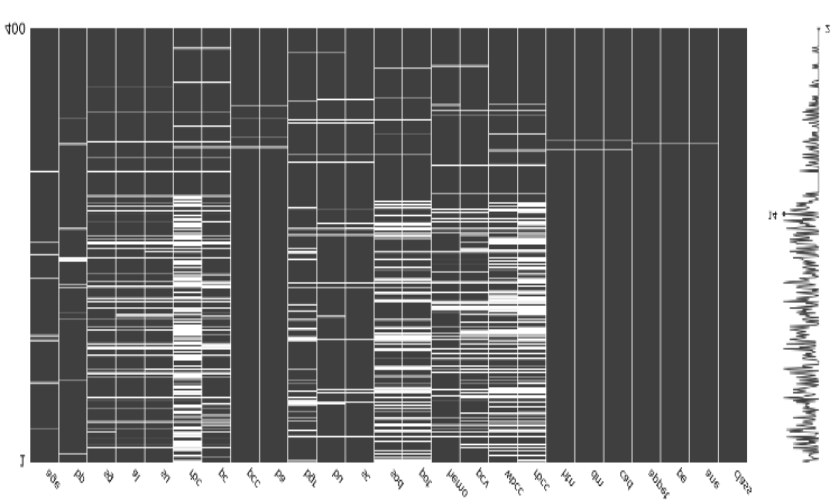

Fig 1: Missing Values

\section{A. Dataset}

The dataset utilized for the prediction of Chronic Kidney Disease has been acquired from the UCI Data Repository [9]. The dataset contains information of 400 individuals with 24 attributes including the class. The Dataset had 250 patients with CKD and 150 patients with NoCKD.

\section{B. Data Preprocessing}

In the preprocessing of the information the missing qualities as shown in Fig 1 were managed by replacing numeric and discrete whole number qualities by attribute mean of the all the instances with a similar class-name as that of the occurrence under consideration and nominal qualities were replaced utilizing attribute mode. Feature Scaling includes rescaling the highlights to such an extent that they have the properties of a standard typical circulation with a mean of zero and a standard deviation of one. For scaling, we utilized the MinMaxScaler [10] from scikit-realize which uses the following condition:

$$
X_{S}=\frac{X-X_{M I N}}{X_{M A X}-X_{M I N}}
$$

\section{Base Classifiers}

\section{- K-Nearest Neighbor}

K-Nearest Neighbors (K-NN) [11] is the classification technique for predicting unidentified samples via looking through the nearest information in design space. K-NN foresees the class by utilizing the Euclidean separation characterized as follows:

$$
d(x, y)=\sqrt{\sum_{i=1}^{k}\left(x_{i}-y_{i}\right)^{2}}
$$

The Euclidean separation $d(x, y)$ in $(2)$ is utilized to quantify the separation for finding the k nearest samples in the example space. The classification of the unidentified sample is recognized by the class of the majority of $\mathrm{k}$ samples.

\section{- Support Vector machine}

Support Vector Machine (SVM) [12] is a directed machine learning design which can be utilized for both regression and classification. In this method, each sample is plotted as a point in n-dimensional space, where $n$ represents the number of features.

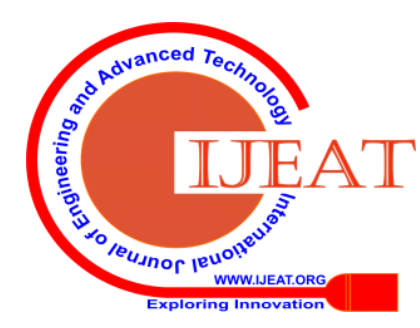


At that point, the classification is performed by finding the hyperplane that separate the two classes quite well. Support Vectors are basically the directions of individual perception.

\section{- Decision Tree}

Decision Tree algorithm [13] is one of the machine learning algorithms used to make prediction based on the decision made by training the dataset. The Decision tree consists of a root, branches and leaves. The feature to be selected as the root node is calculated using the gini index. The attribute having the lowest gini index is taken as the root node and a tree is built by calculating the gini index for other attributes.

\section{- Naïve Bayes}

The Naïve Bayes Algorithm [14] is based on the Bayes theorem given by (3)

$$
P(\text { Class } \mid \text { Data })=\frac{P(\text { Data } \mid \text { Class }) * P(\text { Class })}{P(\text { Data })}
$$

$\mathrm{P}($ Class $)$ is the prior probability and $\mathrm{P}($ Data) is called the evidence. $\mathrm{P}$ (Data| Class) is the Likelihood and P (Class| Data) is the Posterior Probability.

\section{- Logistic Regression}

Logistic Regression [15] is one the basic classification algorithm based on the concept of probability. The logistic regression is the generalized form of linear regression in which the output of the weighted sum of inputs is passed through an activation function called as Sigmoid Function. The activation function maps any real value between 0 and 1 .

\section{- Multilayer Perceptron}

Multilayer perceptron [6] is a kind of feedforward artificial neural network which is a form of a supervised learning technique known as backpropagation for training. The multilayer perceptron consists of three layers namely an input layer, hidden layer and output layer. Each node represents a neuron which makes use of a nonlinear activation function except the input node.

\section{Ensemble Algorithm}

\section{- Majority Voting}

Every base classifier makes a classification for each sample instance. The final output in the majority voting [16] classifier is the one that has obtained more than half of the votes. If the value of prediction in the base classifier is equal then the ensemble method cannot make a stable prediction for that particular sample.

\section{- Weighted Average}

A weighted average algorithm [17] is an extension of a simple averaging ensemble where the contribution of each member to the final prediction is weighted by the performance of the model. The weights of the model are small positive values and the addition of all weights equals one.

\section{- Random Forest}

Random forest algorithm [18] is one of the supervised learning algorithms. The advantage of using random forest is that the algorithm will not overfit the model. The random forest also handles the missing data and it can also be modelled for categorical values.

\section{- ADABoost}

ADABoost [19] is an ensemble algorithm used to boost the performance of the model. It is best used to enhance the performance of week learners. ADABoost algorithm works by increasing the weight of the instance that was wrongly classified and decreasing the weight of the instant that was correctly classified.

\section{- Gradient Boosting}

Gradient Boosting [20] is a supervised machine learning ensemble process used for regression and classification problems. The model is built stage wise and it is generalized by allowing them to optimize a differentiable loss function.

\section{- Bagging}

Bagging known as Bootstrapping Aggregating [20] is an ensemble algorithm used to improve the accuracy and stability of the model. The algorithm avoids overfitting and also reduces the variance.

\section{E. Performance Metrics}

- Confusion Matrix

TABLE I: Confusion Matrix

\begin{tabular}{|c|c|c|c|}
\hline \multicolumn{2}{|c|}{ Confusion Matrix } & \multicolumn{2}{c|}{ Actual Values } \\
\cline { 3 - 4 } & TRUE & FALSE \\
\hline \multirow{2}{*}{ Observed Values } & TRUE & TP & FP \\
\cline { 2 - 4 } & FALSE & FN & TN \\
\hline
\end{tabular}

Table I shows the confusion matrix which is a $\mathrm{K} \mathrm{X} \mathrm{K} \mathrm{matrix,}$ where $\mathrm{K}$ is the number of classes being predicted.

TP (True Positive): The event where the actual value and the predicted value are True.

TN (True Negative): The event where the actual value and the predicted value are False.

FP (False Positive): The event where the actual value is falsely predicted as True.

FN (False Negative): The event where the actual value is falsely predicted as False.

\section{- Accuracy}

It is defined as the quantity of true prediction made as a proportion of all prediction.

$$
\text { Accuracy }=\frac{T P+T N}{(T P+F P+F N+T N)}
$$

\section{- Precision}

Precision is also termed as positively predicted value. It is defined as the ratio of correct positive prediction to the total positively predicted values.

$$
\text { Precision }=\frac{\text { TP }}{\mathrm{TP}+\mathrm{FP}}
$$

Published By:

Blue Eyes Intelligence Engineering

\& Sciences Publication

(c) CoDvriaht: All riahts reserved.

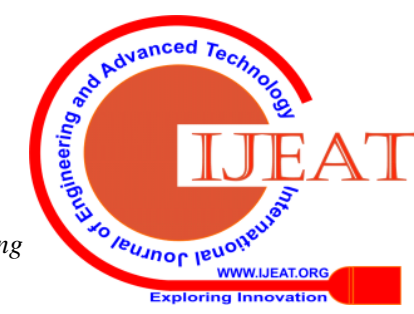




\section{- Recall}

Recall is also termed as Sensitivity, True Positive rate or Probability of Detection.

$$
\text { Recall }=\frac{\text { TP }}{\text { TP }+ \text { FN }}
$$

\section{- AUC-ROC Curve}

ROC (Receiver Operator Characteristic) is a likelihood bend and AUC (Area Under Curve) detect the degree or proportion of detachability. Larger the AUC, better the model is at distinguishing patients with illness and no illness. The ROC curve is plotted with True Positive Rate (TPR) on the $\mathrm{X}$-axis against the False Positive Rate (FPR) on the $\mathrm{y}$-axis.

\section{- Mathew`s Correlation Coefficient (MCC)}

The Matthews correlation coefficient is utilized in Machine Learning as a proportion of the nature of paired and multiclass characterizations. A coefficient of +1 is considered an ideal forecast, 0 a normal irregular expectation and - 1 an opposite forecast. The MCC can be evaluated from the Confusion Matrix using the formula.

$$
\text { MCC }=\frac{(\text { TP } * \text { TN })-(\text { FP } * F N)}{(T P+F P)(T P+F N)(T N+F P)(T N+F N)}
$$

\section{RESULT AND DISCUSSION}

This section of the paper involves the discussion on the individual and ensemble models. First, the performance of individual classifiers on all the 24 features available in the Chronic Kidney Dataset is checked. Next, the performance of ensemble algorithm on all the 24 features is checked. Hyperparameters were tuned to get the best parameters to predict the disease. In both the cases the performance is measured based on accuracy, precision, recall etc. Finally, the performance of individual classifiers and ensembled classifier was compared

\section{A. Individual Classifier Performance on Full Feature}

In this experiment, the performance of six individual classifiers was checked on full features of the dataset. The dataset was split into training set (70\%) and testing set (30\%). The 10-Fold Cross Validation Techniques was applied on the training dataset. The model was fine tuned to get the best performance. Table II shows the performance of the six individual classifiers on full feature and Fig 2 shows the comparison of performance of various individual classifiers.

As shown in table II the performance of Decision Tree was best in comparison to other base classifiers with accuracy $99.16 \%$, precision $98.8 \%$, recall $100 \%$, and the MCC 98.07\%. The Support Vector Machine and the K-Nearest Neighbors (number of neighbors =3) both gave the same accuracy of $98.33 \%$, precision of $100 \%$, recall of $97.59 \%$ and MCC of $96.22 \%$. Naïve Bayes was the weaker performer with accuracy $95.83 \%$, precision $100 \%$, recall of $93.97 \%$ and MCC of $90.98 \%$. The ROC-AUC Curve for the base classifiers are shown in fig 3.
TABLE II: Performance Measure of Various Individua Classifiers

\begin{tabular}{|l|c|c|c|c|c|}
\hline \multicolumn{7}{|c|}{ Base Classifiers } \\
\hline & Accuracy & Precision & Recall & $\begin{array}{l}\text { F1- } \\
\text { Measure }\end{array}$ & MCC \\
\hline $\begin{array}{l}\text { Support } \\
\text { Vector } \\
\text { Machine }\end{array}$ & 98.33 & 100 & 97.5 & 98.78 & 96.22 \\
\hline Naïve Bayes & 95.83 & 100 & 93.97 & 96.89 & 90.98 \\
\hline $\begin{array}{l}\text { Logistic } \\
\text { Regression }\end{array}$ & 98.33 & 100 & 97.59 & 98.78 & 96.22 \\
\hline $\begin{array}{l}\text { Decision } \\
\text { Tree }\end{array}$ & 99.16 & 98.8 & 100 & 99.4 & 98.07 \\
\hline $\begin{array}{l}\text { K-Nearest } \\
\text { Neighbours }\end{array}$ & 98.33 & 100 & 97.59 & 98.79 & 96.22 \\
\hline $\begin{array}{l}\text { Multilayer } \\
\text { Perceptron }\end{array}$ & 97.5 & 100 & 96.38 & 98.15 & 94.44 \\
\hline
\end{tabular}

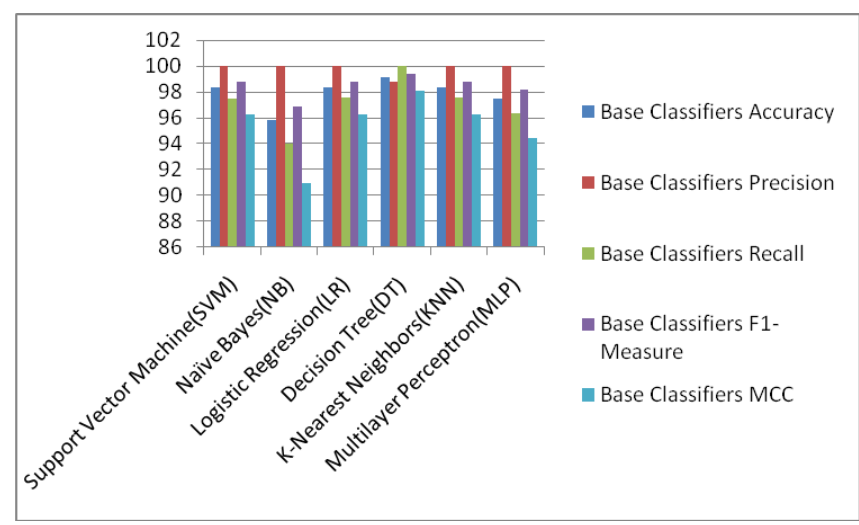

Fig 2: Comparison of Individual Classifiers

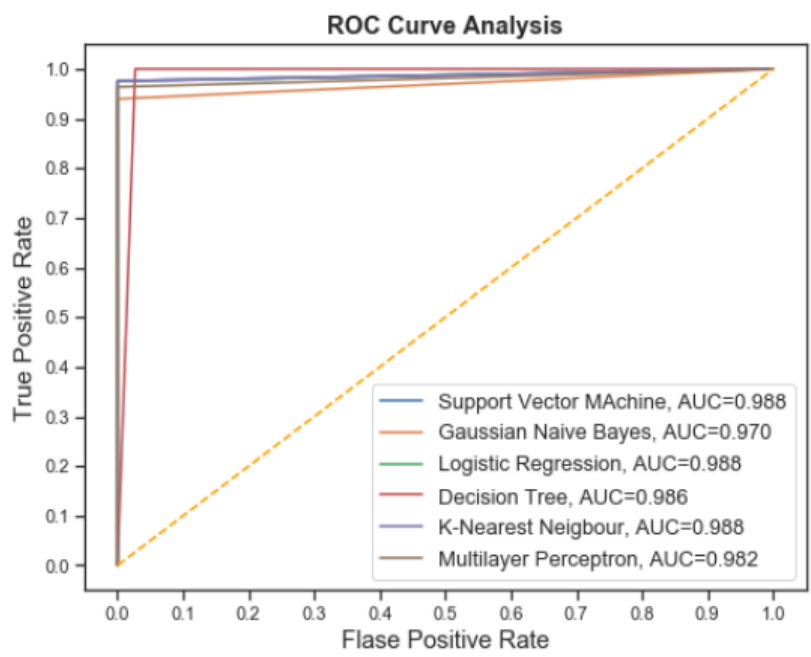

Fig 3: ROC Curve for Base Classifier

As shown in fig 3 the Decision Tree obtained the AUC score of 98.6\%, whereas Support Vector Machine (SVM), K-Nearest Neighbour (K-NN) and Logistic Regression (LR) obtained an AUC score of $98.8 \%$. The Multilayer Perceptron has an AUC score of $98.2 \%$ whereas Naïve Bayes has the lowest AUC of $97 \%$.

\section{B. Ensemble Classifier Performance on Full Feature}

The performance of ensemble classifiers is shown in table III. As shown in table III ADABoost and Random Forest outperformed the remaining classifier with an accuracy, precision,

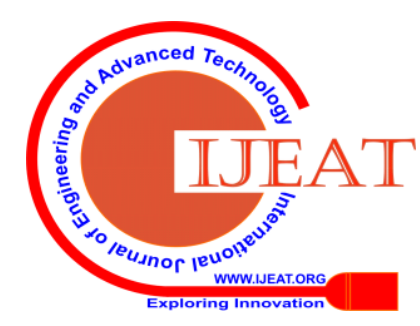


recall and MCC of 100\%. Bagging and Weighted Average classifier performed the second best with accuracy $99.16 \%$, precision $98.8 \%$, recall $100 \%$, and MCC score 98.05\%.

The Majority Voting classifier and Gradient Boosting gave the accuracy of $98.33 \%$, precision of $100 \%$, recall of $97.59 \%$ and MCC of $96.22 \%$. Fig 4 shows the comparison of various ensemble classifiers. The ROC-AUC curve for the ensemble classifier is shown in fig 5 . As shown in fig 5 the ADABoost and Random Forest classifier obtained an AUC of 100\%. The Majority Voting and the Gradient Boosting obtained an AUC of $98.8 \%$ and Weighted Average and Bagging classifier obtained an AUC of 98.6\%.

TABLE III: Performance of Ensemble Classifiers

\begin{tabular}{|l|c|c|c|c|c|}
\hline \multicolumn{7}{|c|}{ Ensemble Algorithm } \\
\hline & $\begin{array}{l}\text { Accurac } \\
\mathbf{y}\end{array}$ & $\begin{array}{l}\text { Precisio } \\
\text { n }\end{array}$ & $\begin{array}{l}\text { Recal } \\
\text { l }\end{array}$ & $\begin{array}{l}\text { F1-Measur } \\
\text { e }\end{array}$ & MCC \\
\hline $\begin{array}{l}\text { Majority } \\
\text { Voting }\end{array}$ & 98.33 & 100 & 97.59 & 98.78 & 96.22 \\
\hline $\begin{array}{l}\text { Weighte } \\
\text { d } \\
\text { Average }\end{array}$ & 99.16 & 98.8 & 100 & 99.4 & 98.05 \\
\hline Bagging & 99.16 & 98.8 & 100 & 99.4 & 98.05 \\
\hline $\begin{array}{l}\text { ADA } \\
\text { Boosting }\end{array}$ & 100 & 100 & 100 & 100 & 100 \\
\hline $\begin{array}{l}\text { Gradient } \\
\text { Boosting }\end{array}$ & 98.33 & 100 & 97.59 & 98.78 & 96.22 \\
\hline $\begin{array}{l}\text { Random } \\
\text { Forest }\end{array}$ & 100 & 100 & 100 & 100 & 100 \\
\hline
\end{tabular}

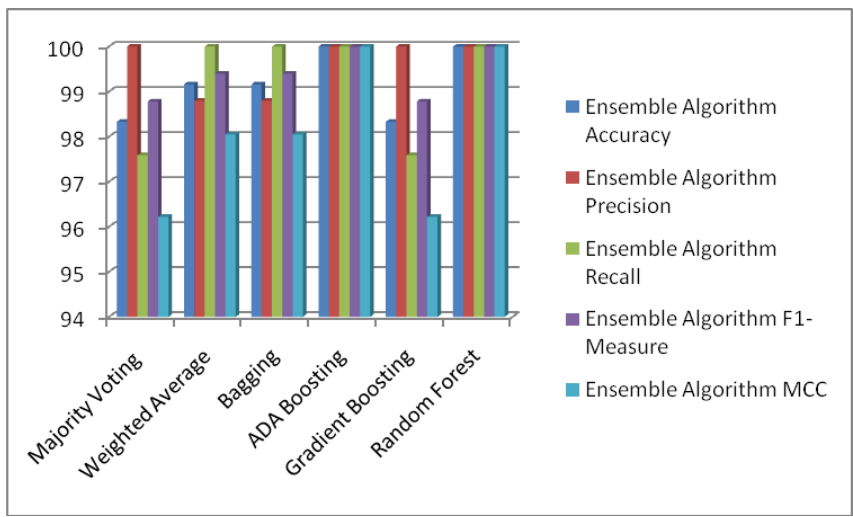

Fig 4: Comparison of various Ensemble Classifiers

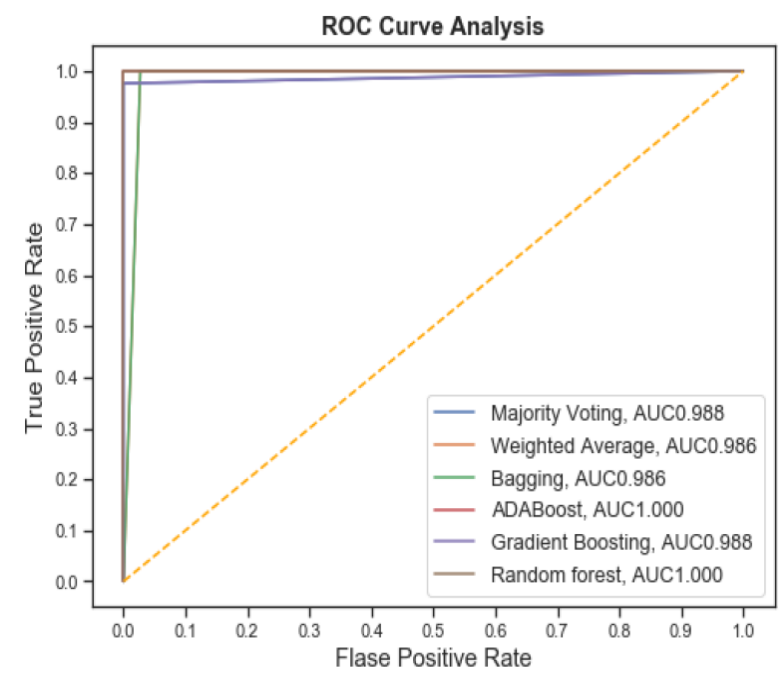

Fig 5: ROC Curve for Ensemble Classifier

\section{CONCLUSION}

The experiment was conducted on the Chronic Kidney Disease dataset. The dataset was tested on six base classifiers like Support Vector Machine (SVM), Logistic Regression (LR), Naïve Bayes (NB), K-Nearest Neighbor (K-NN), Decision Tree (DT) and Multilayer Perceptron (MLP). Next, the dataset was tested on six ensemble classifiers like Majority Voting, Weighted Average, Bagging, ADABoost, Gradient Boosting and Random Forest. The performance in both the cases were measured using accuracy, precision, recall, Area Under Curve (AUC) and Mathew`s Correlation Coefficient. In the base classifier Decision tree performed the best with an accuracy of $99.16 \%$, precision of $98.8 \%$, recall of $100 \%$, MCC of $98.07 \%$ and AUC of $98.6 \%$. In the Ensemble classifier the ADABoost and Random Forest was the best performer with an accuracy, precision, recall, AUC and MCC of $100 \%$. The Ensemble Classifiers performed better than the Base Classifier. The ensemble classifier like Random Forest and ADABoost performed better with 100\% Accuracy, Precision and Recall when compared to the individual classifiers with 99.16\% accuracy, 98.8\% Precision and 100\% Recall obtained from Decision Tree Algorithm. In future, feature selection procedures can likewise be utilized to choose the best features in the datasets. Numerous other individual classifiers and ensemble classifiers can likewise be utilized for forecast of kidney disease with improved accuracy and other performance evaluation metrics.

\section{REFERENCES}

1. "Chronic Kidney Disease," World Kidney Day. [Online]. Available: https://www.worldkidneyday.org/facts/chronic-kidney-disease/. [Accessed: 07-Feb-2020].

2. M. Naghavi et al., "Global, regional, and national age-sex specific mortality for 264 causes of death, 1980-2016: a systematic analysis for the Global Burden of Disease Study 2016," The Lancet, vol. 390, no. 10100, pp. 1151-1210, Sep. 2017, doi: 10.1016/S0140-6736(17)32152-9.

3. "Chronic Kidney Disease (CKD) | NIDDK," National Institute of Diabetes and Digestive and Kidney Diseases. [Online]. Available: https://www.niddk.nih.gov/health-information/kidney-disease/chronic -kidney-disease-ckd..

4. A. Charleonnan, T. Fufaung, T. Niyomwong, W. Chokchueypattanakit, S. Suwannawach, and N. Ninchawee, "Predictive analytics for chronic kidney disease using machine learning techniques," in 2016 Management and Innovation Technology International Conference (MITicon), Bang-San, chonburi, Thailand, 2016, p. MIT-80-MIT-83, doi 10.1109/MITICON.2016.8025242.

5. A. Salekin and J. Stankovic, "Detection of Chronic Kidney Disease and Selecting Important Predictive Attributes," in 2016 IEEE International Conference on Healthcare Informatics (ICHI), 2016, pp. 262-270, doi: 10.1109/ICHI.2016.36.

6. P. Yildirim, "Chronic Kidney Disease Prediction on Imbalanced Data by Multilayer Perceptron: Chronic Kidney Disease Prediction," in 2017 IEEE 41st Annual Computer Software and Applications Conference (COMPSAC), 2017, vol. 2, pp. 193-198, doi: 10.1109/COMPSAC.2017.84.

7. W. H. S. D. Gunarathne, K. D. M. Perera, and K. A. D. C. P. Kahandawaarachchi, "Performance Evaluation on Machine Learning Classification Techniques for Disease Classification and Forecasting through Data Analytics for Chronic Kidney Disease (CKD)," in 2017 IEEE 17th International Conference on Bioinformatics and Bioengineering (BIBE), Washington, DC, 2017, pp. 291-296, doi: 10.1109/BIBE.2017.00-39.

\section{Published By:}

Blue Eyes Intelligence Engineering

\& Sciences Publication

(C) CoDvriaht: All riahts reserved. 
8. J. Xiao et al., "Comparison and development of machine learning tools in the prediction of chronic kidney disease progression," J. Transl. Med., vol. 17, Apr. 2019, doi: 10.1186/s12967-019-1860-0.

9. "UCI Machine Learning Repository: Chronic_Kidney_Disease Data Set." https://archive.ics.uci.edu/ml/datasets/Chronic_Kidney_Disease..

10. F. Pedregosa et al., "Scikit-learn: Machine Learning in Python," $J$ Mach Learn Res, vol. 12, pp. 2825-2830, Nov. 2011.

11. M. Panwar, A. Acharyya, R. A. Shafik, and D. Biswas, "K-nearest neighbor based methodology for accurate diagnosis of diabetes mellitus," in 2016 Sixth International Symposium on Embedded Computing and System Design (ISED), 2016, pp. 132-136, doi: 10.1109/ISED.2016.7977069.

12. D. Selvathi and K. Suganya, "Support Vector Machine Based Method for Automatic Detection of Diabetic Eye Disease using Thermal Images," in 2019 1st International Conference on Innovations in Information and Communication Technology (ICIICT), 2019, pp. 1-6, doi: 10.1109/ICIICT1.2019.8741450.

13. "Decision Trees for the Detection of Skin Lesion Patterns in Lower Limbs Ulcers - IEEE Conference Publication." [Online]. Available: https://ieeexplore.ieee.org/document/7881426.

14. R. Devika, S. V. Avilala, and V. Subramaniyaswamy, "Comparative Study of Classifier for Chronic Kidney Disease prediction using Naive Bayes, KNN and Random Forest," in 2019 3rd International Conference on Computing Methodologies and Communication (ICCMC), 2019, pp. 679-684, doi: 10.1109/ICCMC.2019.8819654.

15. L. Liu, "Research on Logistic Regression Algorithm of Breast Cancer Diagnose Data by Machine Learning," in 2018 International Conference on Robots Intelligent System (ICRIS), 2018, pp. 157-160, doi: 10.1109/ICRIS.2018.00049.

16. R. Atallah and A. Al-Mousa, "Heart Disease Detection Using Machine Learning Majority Voting Ensemble Method," in 2019 2nd International Conference on new Trends in Computing Sciences (ICTCS), 2019, pp. 1-6, doi: 10.1109/ICTCS.2019.8923053.

17. K. Shashvat, R. Basu, A. Bhondekar, and A. Kaur, "A Weighted Ensemble Model for Prediction of Infectious Diseases," Curr. Pharm. Biotechnol., vol. 20, Jun. 2019, doi $10.2174 / 1389201020666190612160631$.

18. J. V. Eyck et al., "Prediction of Chronic Kidney Disease Using Random Forest Machine Learning Algorithm," 2016.

19. H. Rajaguru and S. K. Prabhakar, "Analysis of adaboost classifier from compressed EEG features for epilepsy detection," in 2017 International Conference on Computing Methodologies and Communication (ICCMC), 2017, pp. 981-984, doi: 10.1109/ICCMC.2017.8282614

20. X. Yuan et al., "A High Accuracy Integrated Bagging-Fuzzy-GBDT Prediction Algorithm for Heart Disease Diagnosis, " in 2019 IEEE/CIC International Conference on Communications in China (ICCC), 2019, pp. 467-471, doi: 10.1109/ICCChina.2019.8855897.

\section{AUTHORS PROFILE}

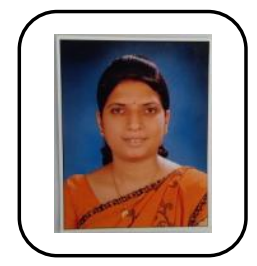

Nikhila $\mathbf{G}$ is currently a $2^{\text {nd }}$ year $M$. Tech DECS Student in the Department of Electronics and Communication Engineering at Center of PG Studies VTU, Mysore. She has received her B.E Degree in Telecommunication from P.A. College of Engineering, Mangalore. She has a teaching experience of 11 years in the Department of Electronics and Communication Engineering, Mangalore Institute of Technology and Engineering, Moodabidri. Her research interest includes Computer Networks, Embedded Systems, Machine Learning, IOT.

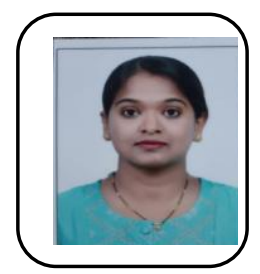

Meghashree A C is working as an Assistant Professor in the Department of Electronics and Communication Engineering, VTU, Centre of PG Studies, Mysore. Her research interest includes Embedded Systems, IOT, Machine Learning and Wireless Sensor Network.

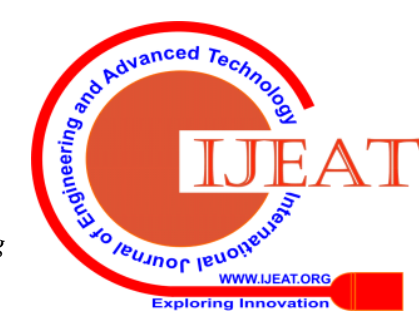

